\title{
HUMAN-ROBOT SYNERGY FOR COOPERATIVE ROBOTS
}

\author{
$U D C(004.896: 61): 681.5 .01$
}

\section{Maria Kyrarini ${ }^{1}$, Adrian Leu ${ }^{1}$, Danijela Ristić-Durrant ${ }^{1}$, Axel Gräser1, Anja Jackowski ${ }^{2}$, Marion Gebhard ${ }^{2}$, Jochen Nelles ${ }^{3}$, Christina Bröhl $^{3}$, Christopher Brandl ${ }^{3}$, Alexander Mertens ${ }^{3}$, Christopher M. Schlick ${ }^{3}$}

\author{
${ }^{1}$ Institute of Automation (IAT), University of Bremen, Germany \\ ${ }^{2}$ Sensors and Actuators in Medical Engineering (SAM), University of Applied Sciences, \\ Westphalia Gelsenkirchen, Germany \\ ${ }^{3}$ Institute of Industrial Engineering and Ergonomics, RWTH Aachen University, Germany
}

\begin{abstract}
This paper presents two human-robot cooperative application scenarios of the project MeRoSy (Human-Robot Synergy) funded by the German Federal Ministry of Education and Research. The first scenario relates to the human-robot cooperation in an industrial application, while the second one refers to the robotic workplace assistance for people with disabilities. The presented scenarios reflect different aspects of human-robot interaction, among others different novel possibilities for human-robot interaction depending on different physical abilities of human co-worker. Beside the consideration of the human-robot cooperative technologies in two MeRoSy scenarios, this paper considers also the identification and classification of the ethical, legal and social implications (ELSI) in the context of human-robot cooperation.
\end{abstract}

Key words: human-robot interaction, human-robot collaboration, cooperative robots, assistive robots, ELSI (ethical, legal and social implications)

\section{INTRODUCTION}

There is growing interest in the applications in which humans and robots are working together. Whereas in the past it was all about replacing the work of humans with that of robots, today the focus is much more on the robot as an assistant or co-worker. Due to the progress in research and development in the robotics and artificial intelligence, robots became capable and able to perform number of tasks autonomously without human

Received December 01, 2016

Corresponding author: Maria Kyrarini

Institute of Automation (IAT), University of Bremen, Germany

E-mail: mkyrar@iat.uni-bremen.de 
intervention. However, in spite of this progress, there are still some limits to the abilities of an autonomous robot. Further, some tasks that are difficult for a robot may be easy for a human (for example cognitive reasoning, recognition of objects newly appeared in the scene, and manipulation of very small objects). Similarly, some tasks that are difficult or tedious for a human are easy for a robot (for example, performing mathematical computations and performing precise repeated movements with a short cycle time). This has indicated the need for research in synergizing the human and robot's abilities to perform a task efficiently. For example, in [1], the synergetic work of the assistive robot FRIEND and the end-user, a person with disability, was described, where the robot supports the end-user working on a regular basis at a library workplace. It was shown that a success rate of $82 \%$ was reached for fully autonomous task execution, while an increase of the overall success rate to $95 \%$ was achieved when the FRIEND system and the end-user were working together.

[1] represents the research results of one of the groups that have been working in the field of assistive robotics, where robots assists humans in performing different tasks without direct physical human-robot interaction (HRI). Beside assistive robotics, in last decades there has been also a significant work in human-robot cooperation in wearable robotics, where robots in direct physical HRI support humans in performing different movements [2]. In contrast to assistive and wearable robotics, the human-robot synergy in context of robotic workplace assistance for human worker is quite a new research topic. Namely, robots and humans, for decades kept separate from each other on factory floors, are moving slowly toward integration. After years of separating robots from humans by putting them into safety cages to ensure human safety, some companies are finding ways to put them alongside people. For example, Daimler and KUKA signed strategic cooperation [3] for the partnership that focuses on humanrobot cooperation to achieve optimizations both for production workers and in manufacturing processes. The collaborative robots have been tested for different tasks such as assembly tasks.

With robots increasingly being used on factory floors as well as in different non-industrial applications such as assistive and rehabilitation robotic applications, researchers are looking for ways to help humans work better with their electromechanical counterparts. At the first place, a lot of robotics research focuses on developing safety strategies to make sure robots and humans can operate safely side by side [4]. However, safety strategies are not enough. To make robots smart and flexible enough to work effectively with people it is necessary to progress in different robotic technologies and abilities. These advances concern among others robot's perception capabilities, human-robot interface, robot's learning capabilities [5][6]. Also, robots and people working at the same time in the immediate proximity while performing together a work task has brought up many ethical, legal and social implications (ELSI), which represent an important research topic in the robotic community.

The project MeRoSy [7] funded by the German Federal Ministry of Education and Research, aims at advancing the state-of-the-art in the research of human-robot synergy for cooperative robots. There are two MeRoSy working scenarios: robot's cooperative work in a library scenario with a person with disability and robot's cooperative work with an able bodied person in an industrial assembly scenario. These two scenarios have been chosen so to reflect different aspects of human-robot interaction, among others different possibilities for human-robot interaction depending on different physical abilities of human co-worker. This paper presents an overview of the first results achieved towards building the humanrobot collaboration in both scenarios, which are presented together with the initial consideration of related ELSI aspects. 


\section{HUMAN-ROBOT COOPERATION IN AN INDUSTRIAL ASSEMBLY SCENARIO}

An assembly industrial scenario represents a good example of a robotic application which brings a benefit through the human-robot cooperative work. In the MeRoSy project, the scenario of the assembly of the robot gripper in the human cooperative work with the twoarmed robot, the pi4 Workerbot [8], is considered. Bearing in mind that the robot's gripper contains a number of very small parts, such as screws, the full automation of assembly process is still not possible as the robot manipulator does not have human level of dexterity. On the other hand, cooperation of the human worker with the robot can significantly increase the effectiveness of the assembly process by using the robot's advantages of, for example, high speed and possibility for repetition of tedious tasks. The starting point in the MeRoSy scenario has been division of the object manipulation tasks between human and robot co-workers so that different parts of the gripper to be assembled are manipulated by the robot and different parts are manipulated by the human co-worker. This division of object manipulation tasks was done bearing in mind advantages and disadvantages of co-workers, the robot and the human, in order to achieve higher effectiveness. In contrast to human skilled co-worker, who has experience and cognitive skills of manipulating the objects autonomously, the robot has to be firstly taught on how to perform the object manipulation. In the following, the first results on developing and implementing learning framework to enable robot to learn from the human demonstrations and then to reproduce the learned skills are presented.

\subsection{Robot learning framework for object manipulation tasks}

A robot learning framework is proposed in the MeRoSy project to enable robots to learn skills, which are needed to perform particular object manipulation tasks, from human demonstrations. The overall structure of this framework is shown in Fig. 1.

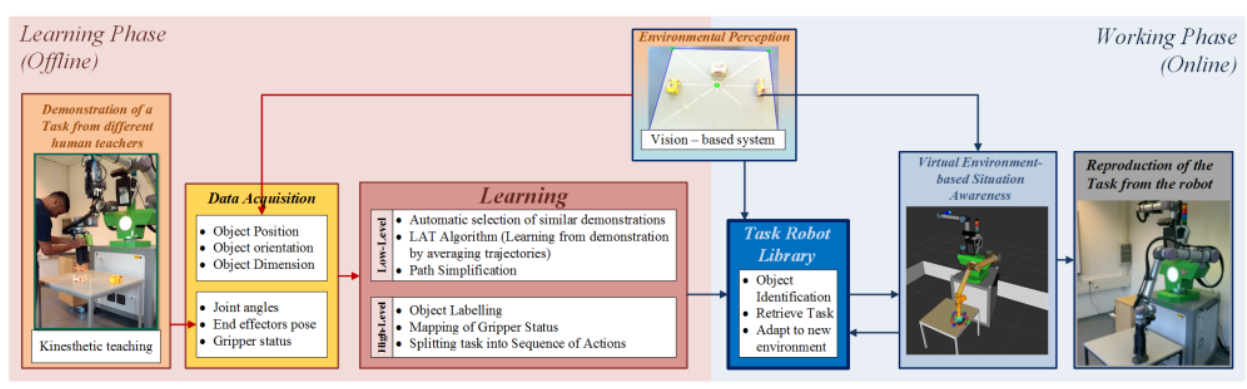

Fig. 1 Block-diagram of the robot learning framework in human-robot cooperation in the assembly scenario

The idea behind the proposed learning framework is that a human "teacher" demonstrates the actions needed to complete the task to the robot learner and the robot learner imitates them. One popular method for demonstration is the kinesthetic teaching [9][10], where the teacher guides physically the robot's end effector through the task and the robot movements are recorded by the robot's sensors. Kinesthetic teaching can work only for robots driven by zeroforce controllers [11], which is the case of the used pi4 Workerbot. In order to perform autonomously the object manipulation, the robot has to learn skills enabling the following actions: "grasping of an object", ,moving along the demonstrated trajectory from the grasping 
to the target (releasing) position while carrying the object“" and ,releasing the object at the desired target position". The working hypothesis is that the learning from one human teacher has limitations as the teacher may make mistakes during the demonstration that the robot will be vulnerable to. The human teacher is also slower than the robot, has a low precision compared to the robot and may perform unnecessary movements in attempts to be very precise in positioning the end-effector. Also, as the different human demonstrations lead to differently demonstrated skills, an optimally learned skill could outcome from a combination of different demonstrations. This is particularly important in a human-robot collaborative work in an industrial scenario where the robot could work with different human co-workers. Because of this, in the present work, learning from multi-persons demonstrations is suggested. The presented learning framework consists of the following modules.

\section{A. Demonstration of the task from different humans}

Several persons are asked to teach the robot the skill of „moving along the trajectory from the start to the target position". The persons are asked to move the robot arm, which is in zero-force control mode, by holding the gripper so that the gripper is moved from one point to another on the working-table with the objects to be manipulated placed on it. Each person is asked to demonstrate the skill twice.

\section{B. Environmental Perception}

The Environment perception module is based on 3D-point cloud based processing [12] to extract the information on position, orientation and dimensions of every object in the view of the robot vision-based perception system. In the presented MeRoSy working scenario, a table with the objects placed on it is in the view of the used Kinect2 [13] camera.

\section{Data Acquisition}

This module stores in the database all the data provided by Environmental Perception Module. In addition, the Data Acquisition module records and stores in the robot's database the joint angles and the end effector pose of the robot arm as well as the actuation status of the gripper ("On" denoting the activated gripping status and "Off" denoting not-activated gripping status) during the human demonstrations of the task

\section{Learning}

The learning module consists of the low-level learning of the skill "moving along the demonstrated trajectory from the grasping to the target (releasing) position" and highlevel learning of sequence of actions needed to perform object manipulation task.

\section{D1. Low-level Learning}

The low-level learning consists of the following steps: Automatic selection of similar demonstrations, learning from demonstrations by Averaging Trajectories (LAT) algorithm [14] and path simplification.

- Automatic selection of similar demonstrations

In the following, this low-level learning step is explained on example of human demonstrations performed by 5 persons, human teachers, where each demonstrator performed two demonstrations. While the human teachers were performing the demonstrations, the question raised: "Should all 10 demonstrations be used to generate the learned skill?" Some demonstrated trajectories (as shown in Fig.2) differ a lot from each other although the start and the end point of the trajectories are almost the same. In order to select the trajectories that are 
the most similar and to filter out the outlier trajectories, a novel automatic Dynamic Time Warping (DTW)-based algorithm is implemented.

During the considered multi-persons demonstrations of moving an object from one point to another on the work-table, the Cartesian coordinates of the gripper tip were recorded. The recorded datasets had different number of samples, since every human demonstrator performed the task with different speed, as can be seen in Fig.2. In order to have the same number of samples for every dataset, resampling was needed for which the Hermite extrapolation [15] was used. Fig. 3 shows the datasets of the gripper (end effector) position from the 10 demonstrations after extrapolation. Dynamic Time Warping (DTW) [16][17] is a method to find an optimal alignment between two given time-series which may vary in speed and time and to measure the similarity of these two time-series. The smallest the DTW distance is, more similar the two time-series are.

In presented approach for the selection of similar demonstrations, firstly, the DTW distance in 3D is calculated as follows:

$$
D T W_{3 D}(i, j)=D T W_{x}(i, j)+D T W_{y}(i, j)+D T W_{z}(i, j) .
$$

Where $D T W_{x}(i, j)$ is the DTW distance between the demonstrations $i$ and $j$ in X-axis, where $i, j \in\{1,2, \ldots, N\}$. Similarly, $D T W_{y}(i, j)$ and $D T W_{z}(i, j)$ are the DTW distances between the demonstrations $i$ and $j$ in $\mathrm{Y}$-axis and Z-axis, respectively. $N$ is the number of demonstrations (in the presented case 10).

Secondly, the so-called "reference" demonstration which is the most similar to the rest demonstrations is selected. The similarity vector between each trajectory and the rest trajectories is calculated as follows:

$$
\operatorname{similarity}(i)=\sum_{j=1}^{N} D T W_{3 D}(i, j), \forall i \in\{1,2, \ldots, N\}
$$

The demonstration that has the minimum value in vector similarity is the "reference" demonstration and it is denoted with $r$. In the presented case, the demonstration 4 was the one with the minimum value. After deciding on the "reference" demonstration it is needed to find the demonstrations which are similar to the "reference" demonstration. For this a threshold equal to 1 was defined by the user, in order to get the trajectories with more similarity. The demonstrations that satisfy the inequality (3) are the demonstrations that are similar to the "reference" demonstration. In the presented case the demonstrations 5, 9 and 10 were those satisfying the inequality (3).

$$
D T W_{3 D}(r, j)<1, \forall j \in\{1,2, \ldots, N\} .
$$

The four demonstrations (demo 4, demo 5, demo 9, demo 10) were selected demonstrations to be used for the learning.

- Learning from demonstrations by Averaging Trajectories (LAT)

From the selected similar demonstrations (demo 4, demo 5, demo 9, demo 10), one path is generated using the so-called LAT method (Learning from demonstration by Averaging Trajectories) [14]. This path is considered as the learned path by the robot and can be seen in Fig. 4. 
- Path Simplification

The Ramer-Douglas-Peucker Algorithm [18][19] is a polyline simplification algorithm that is used in cartography [20]. This method is used to automatically select and save only important "key-points" of the learned path in the database. The original learned path and the way-points of the simplified path are shown in Fig. 5. The advantage of saving only important "key - points" instead of the whole path is that it is faster to search a database with less points in order to retrieve an already learned path or a segment of it for the purpose of its adaptation to a specific environment and its reproduction. The simplified path is considered as "learned path" to be used further in high-level learning step.
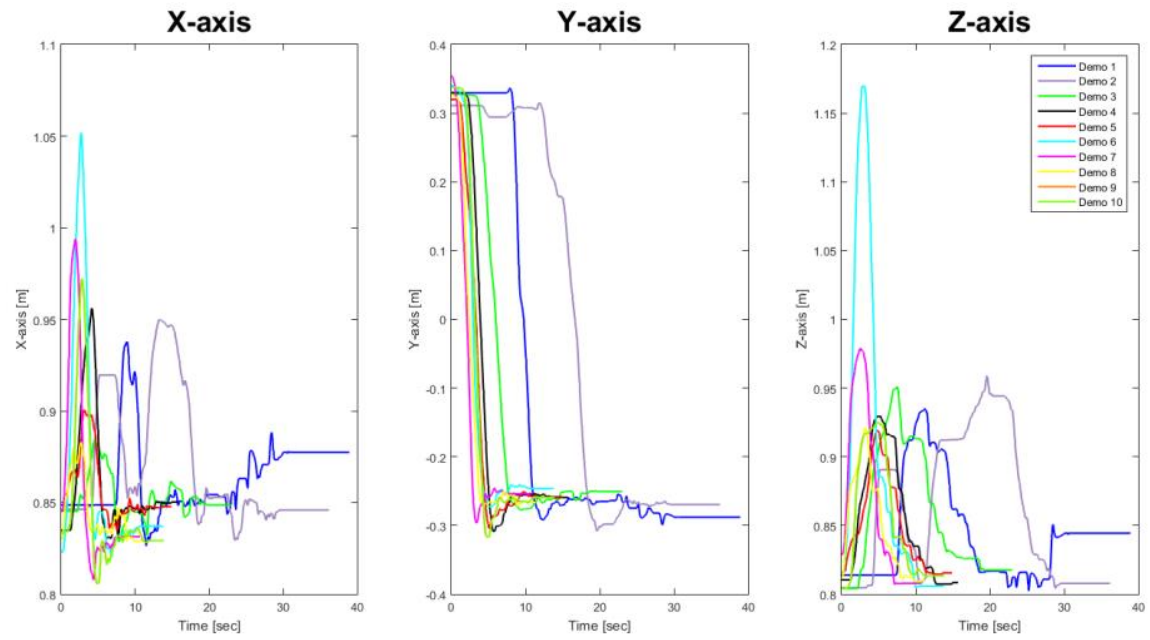

Fig. 2 Recorded gripper positions $(\mathrm{X}, \mathrm{Y}, \mathrm{Z})$ from 10 different demonstrations during kinesthetic teaching
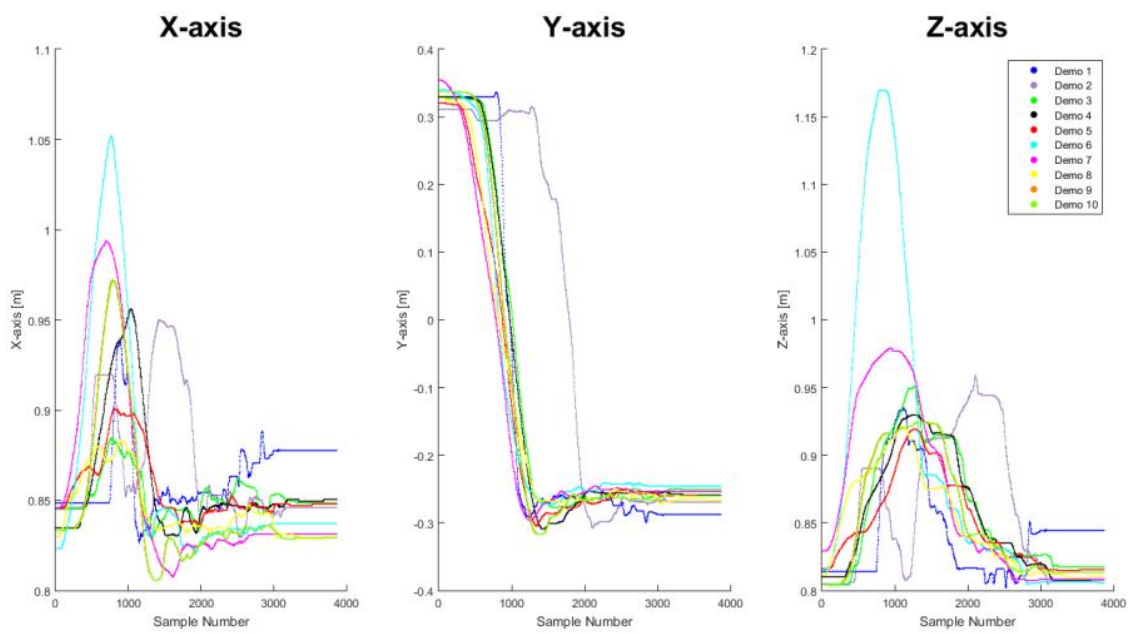

Fig. 3 Gripper positions $(\mathrm{X}, \mathrm{Y}, \mathrm{Z})$ for 10 different demonstrations after extrapolation 

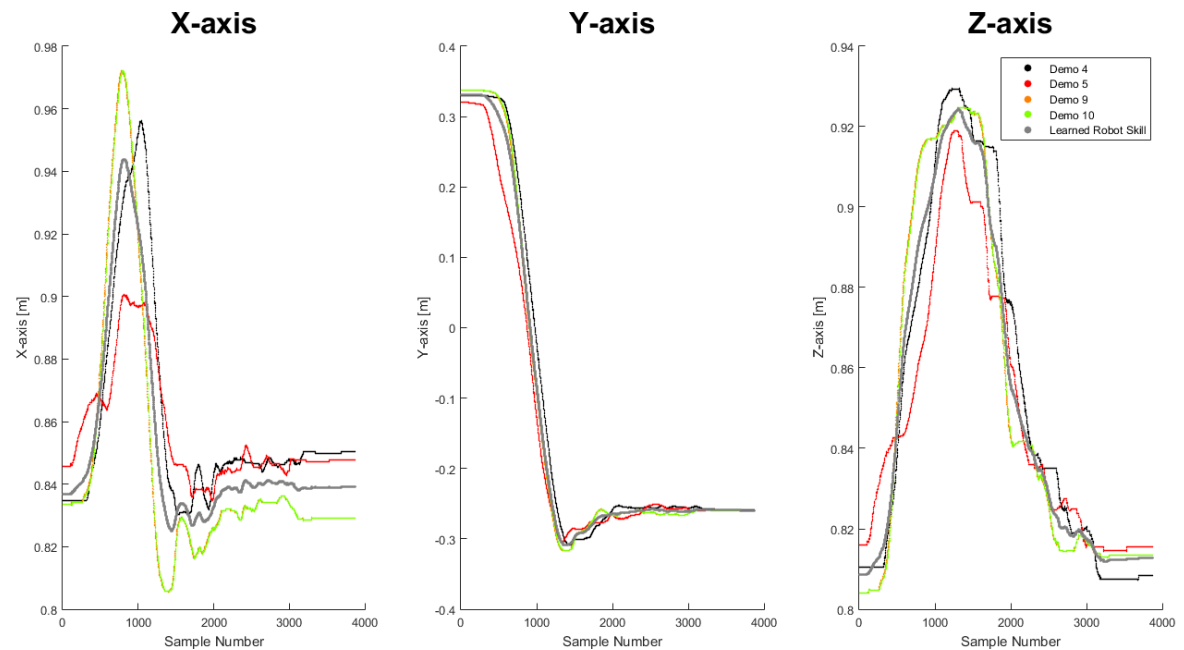

Fig. 4 Automatic selection of similar demonstrations and learned robot skill

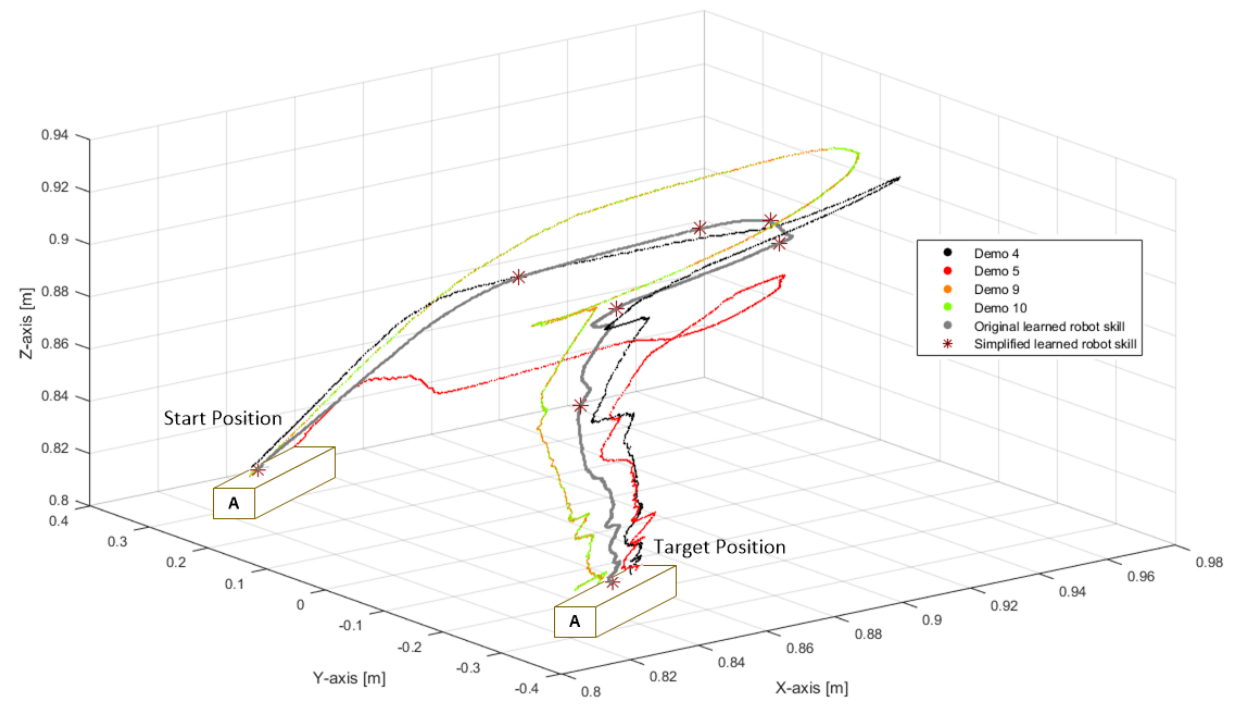

Fig. 5 3D-Representation of the demonstrations selected automatically, of the original learned robot skill and simplified (learned) path for the learned robot skill

\section{D2. High-level Learning}

The high-level learning of the task consists of three steps: labelling of the objects, mapping of the gripper status to the learned path and splitting of the task into sequence of actions.

- Labelling of objects

During the demonstration phase, the objects involved in the task are labeled with specific IDs which denote the position of the gripper when the gripper actuation status was $\mathrm{ON}$ and 
the robot arm (left or right) which was used for object grasping. For example, the ID "left_pick_l" means that the first identified object was picked with the left arm. This labeling method indicates also the necessary sequence of actions for the manipulation task, as the objects to be manipulated are ordered as indicated by the ID.

- Mapping of the gripper status onto the learned path

The Cartesian pose of the robot's end-effector (gripper) for the positions when the robot grasped and released an object is compared with the learned path waypoints and the closest waypoint is labeled as skill point, that is "grasping" or "releasing" point.

- Splitting Task into actions

After the mapping of gripper actuation status to the learned path, the learned path is split into actions based on the low-level learned skills. The robot learns the sequence of actions of the task (high-level) also including the action of following the path that needs to be followed in order to perform those actions (low-level). In the considered example task, the robot learns the following sequence of actions: grasp the object with ID "left_pick_l", move it along the learned path and release it at a specific learned "releasing" position.

\section{E. Task Robot Library (TRL)}

The Task Robot Library (TRL) module is responsible for the identification of the objects based on their dimensions and for assignment of the IDs, for retrieving the task based on the identified objects and, at the end, for the adaptation of the learned path if the environmental conditions have been changed. Firstly, the current working environment is perceived by the "environmental perception" module. The TRL compares then the number of perceived objects and their dimensions with the objects identified during the demonstration phase in order to assign IDs to currently perceived objects and to retrieve the task. The TRL checks if the current environment (position and orientation of the perceived objects) is the same as the environment during the demonstration phase. If it is the same, the same IDs are assigned to current objects and then the task is reproduced by the robot. However, if the position of a current object differs from the demonstrated position, TRL module will send request to the "Virtual environment-based representation of the robot awareness" module to update the grasping position of the object so that the robot can perform the learned task of grasping the object, moving it along the learned path and releasing it at learned "releasing" position. Additionally, if the Task Robot Library Module detects that there are additional objects that were not present during the demonstrations, then those objects are considered as obstacles. If an obstacle is located along the learned path, TRL will remove those collision waypoints from the learned path and it will request from the "Virtual environment-based representation of the robot awareness" module to control the robot so to avoid the obstacle and to continue robot movement so that robot's end-effector moves along the remained part of the learned path. If the perceived objects are completely different from those present during demonstrations the TRL requests additional robot's training.

\section{F. Virtual environment-based representation of the robot awareness}

A virtual environment has been developed using the ROS-based tool rviz (ROS visualization) [21] to illustrate the robot environmental awareness and the learned task that robot shall perform in real-time. In Fig. 6(a), the virtual environment of the robot in its home pose can be seen together with the table in front of the robot and a object to be manipulated, which is placed on the table. The position of the table with respect to the 
world coordinate system, as well as the position of the object on the table, is obtained by the Environment Perception Module. If the current position and orientation of the object is such that the previously learned grasping point is different from the current position and orientation of the object, the Task Robot Library (TRL) requests the correction of the position using motion planning from MoveIt [22]. Similarly, if there are obstacles along the learned path, the path re-calculated for the purpose of obstacle avoidance can be viewed in the virtual environment before being executed by the robot so that undesired robot movements could be avoided. The resulted object grasping, after robot performed movement along learned/re-calculated path, can be seen in Fig. 6, both as awareness representation in the virtual environment (b) and as the real-world image (c).

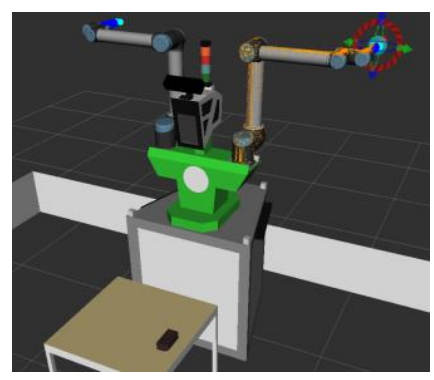

(a)

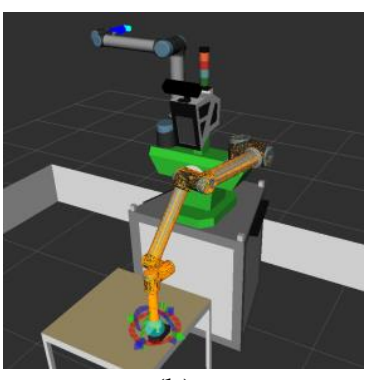

(b)

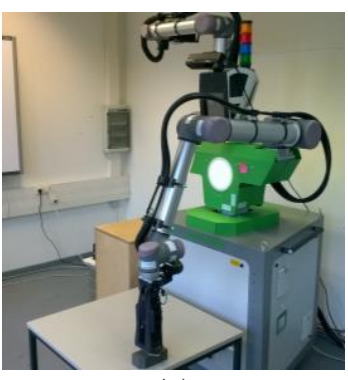

(c)

Fig. 6 Object grasping. (a) Robot home pose, (b) Virtual environment, (c) Real-world scene

\section{G. Reproduction of the Task by the Robot}

After the execution of the learned task is visualized by Virtual environment-based representation of the robot awareness module and after the human co-worker approves the execution through the graphical user interface (GUI), the robot performs the learned task. Fig. 7

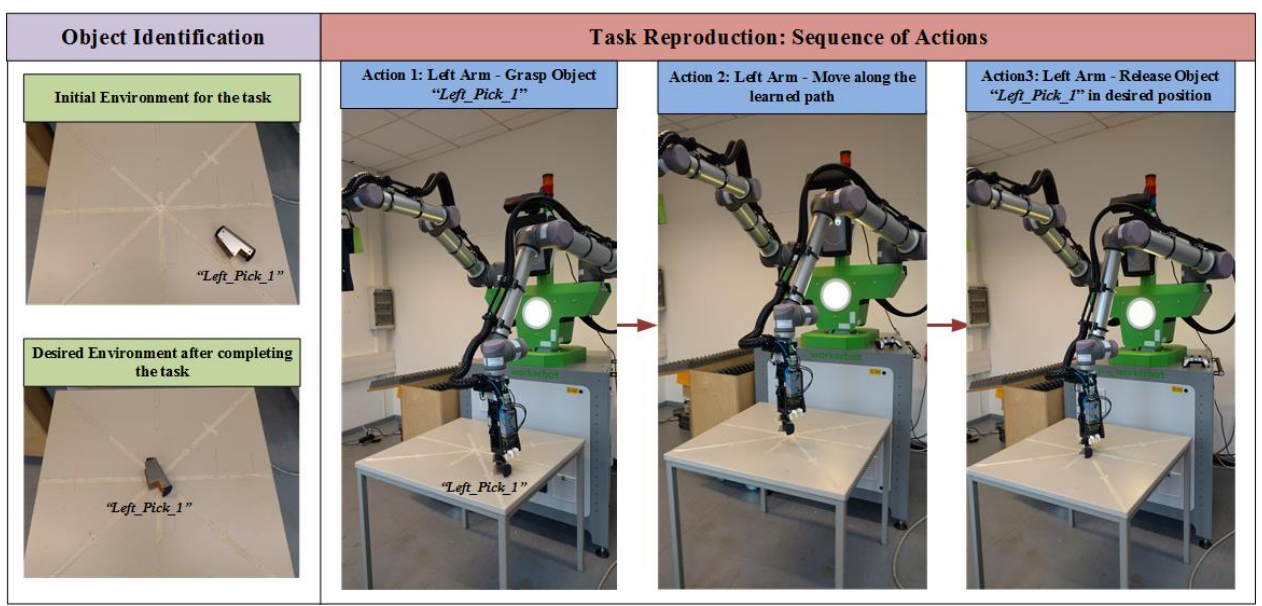

Fig. 7 Object Identification and task reproduction by the robot in the case of the same environmental conditions as during demonstrations 
illustrates the object identification and the sequence of the actions needed for the execution of the learned manipulation task in the case of the environment which is the same to the environment during the demonstrations. Fig. 8 presents the robot ability to adapt the learned path to different environmental conditions. The initial position of the "Left_Pick_1" object is different from one shown in Fig. 7 and additionally an obstacle has been added. As it can be seen from Fig. 8, the robot is able to perform successfully the task and to avoid collision, without any additional input from the human co-worker.

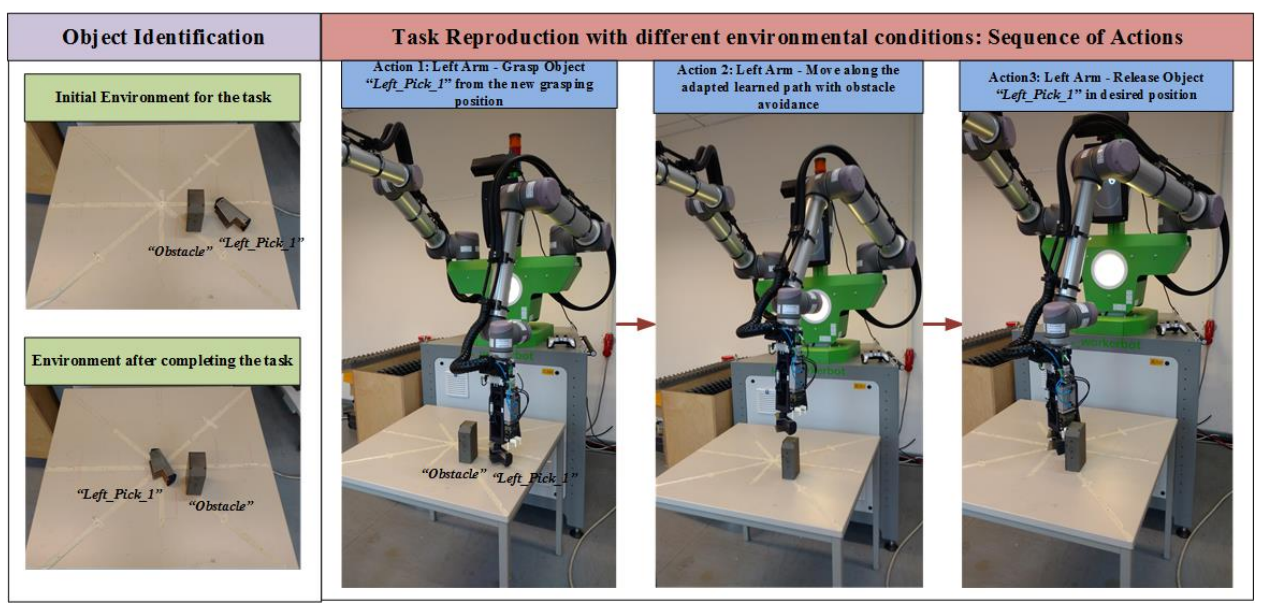

Fig. 8 Object Identification and task reproduction by the robot in the case of the environmental different from the conditions during demonstrations

\section{HUMAN-ROBOT COOPERATION IN AN ASSISTIVE ROBOTIC SCENARIO}

The aim of this human-robot cooperation is to demonstrate an opportunity for disabled people to work with the help of an assistive robot at a workspace in a library. In the framework of MeRoSy, a novel human-robot interaction based on head motion and head gestures, captured with an inertial measurement unit, has been developed. People with a loss of the upper limbs motor functions, e.g. due to diseases such as multiple sclerosis, amyotrophic lateral sclerosis or traumatic injuries, often have to retire from working life because they do not meet the needed physical abilities anymore. Assistive robots enable an interaction with their environment and therefore can increase autonomy of these people. For the presented hands-free human-robot interaction in the library scenario, the end-user, that is the human co-worker, controls a semi-autonomous assistive robot with head motion.

\subsection{Assistive robot in a library scenario}

The considered assistive robot scenario is shown in Fig. 9. It takes place in a library, where the human co-worker's task is to catalogue books with the help of an assistive robot. Therefore, the following actions are necessary to be performed in human-robot cooperation: 
1. Move the gripper to the bookshelf

2. Take a book

3. Move the book to the table

4. Place the book in front of the human co-worker

5. Open the book

6. Catalogue the book

7. Close the book

8. Take the book from the table

9. Move the book to another bookshelf

10. Place the book on the bookshelf
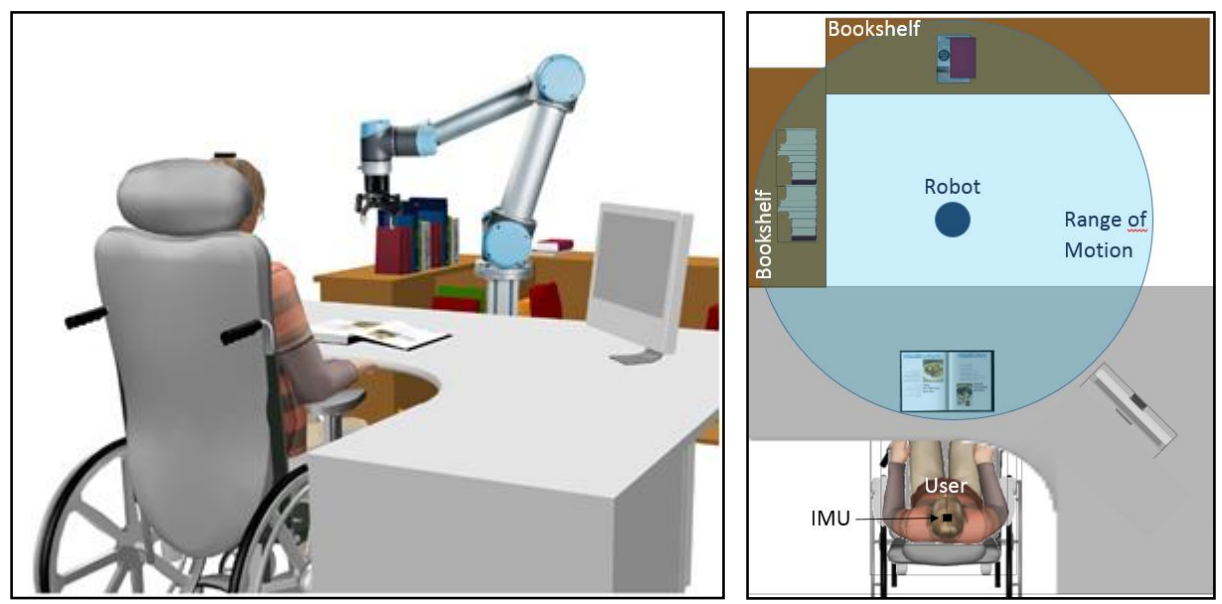

Fig. 9 Library scenario as three-dimensional view (left) and as view from above (right).

After completing the above sequence of actions in order to catalogue the next book, the human co-worker initialises the new sequence of actions starting from the first one.

The human co-worker's safety is guaranteed due to the safety distance between the user and the range of motion of the robot. Even if the robot is stretched to its maximum length, it cannot reach the human co-worker, as it is illustrated in Fig. 9. Furthermore, the human co-worker has always the possibility to intervene and can control the robot's movements directly with the motion of his head.

\subsection{Head motion based robot control}

Within the library scenario of the MeRoSy project, the target end-users are disabled people who cannot use their hands. Thus, the proposed human-robot interface uses head motions as input signals.

\subsubsection{Interaction technology: IMU}

In the assistive robotic scenario, human co-worker's head motion is captured with an inertial measurement unit (IMU) and it is to be used for the control of the robot arm. IMUs are 
small, cheap, energy-efficient, self-contained and allow a precise motion measurement, so they are suitable for human-robot interfaces. The head motion is acquired with the sensor module FSM-9 by Hillcrest Laboratories [23]. This nine-axis inertial measurement unit includes three accelerometers, three gyroscopes, three magnetometers and on-board signal processing software. The raw data of the single sensors is calibrated and fused in the signal processing software, so the sensor orientation is directly given as sensor output.

The controlled robot arm is the six degrees of freedom (DOF) robot UR5 of Universal Robots [24] with the adaptive gripper 2-Finger Robotiq 85 [25]. A 27-inch screen is used to show the graphical user interface (GUI) and to give visual feedback to the human co-worker.

\subsubsection{Interaction Design: Control structure and interfaces}

The human head has three degrees of freedom (DOFs). The robot arm has six DOFs and the gripper provides the seventh DOF to be controlled. To control these seven DOFs intuitively with the head motions, the robot's movements are divided into four control groups, as shown in Fig. 10 [26].

Within the control groups, the user controls the velocity of the robot, as suggested in [27]. To switch between the different groups, switching commands that differ from the signals for direct control have to be defined. In the framework of MeRoSy, head gestures are proposed as switching commands. Head gestures are particular movements, which the user performs with the head. The gestures are recognized with an analytical algorithm [28].

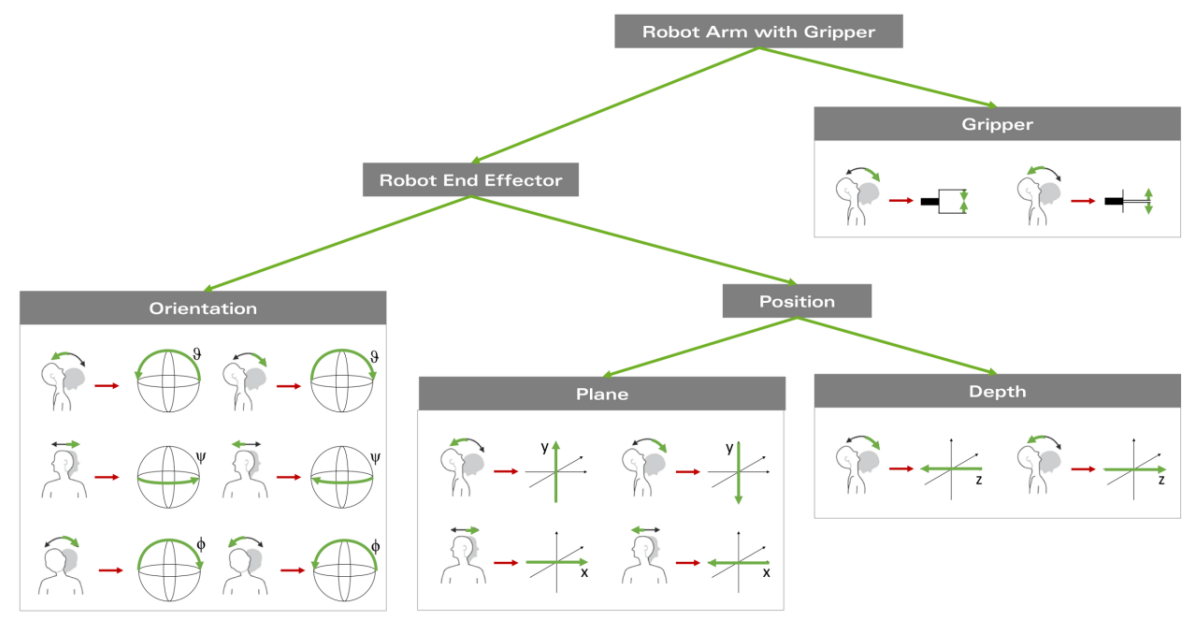

Fig. 10 Control structure to map head motion with three DOFs to motion of robot arm and gripper with seven DOFs [26]

Two control interfaces based on the head motion are used for direct robot control within MeRoSy:

1. GUI-Aided Interface

The first interface is based on GUI-aided two-stage parallel switching [29]. In the first stage, the end-user controls a mouse cursor on the screen with head motions. The different robot control groups can be chosen by activating a corresponding 
button on the GUI. The system switches to the second stage robot control. To stop robot control and return to the control of the mouse cursor, the end-user has to perform a head gesture.

2. Gesture Based Interface

The second interface uses four head gestures as switching commands [30]. Robot control is activated and deactivated with the performance of a gesture. Moreover, the end-user can switch immediately between the different control groups using different gestures (Fig. 11).

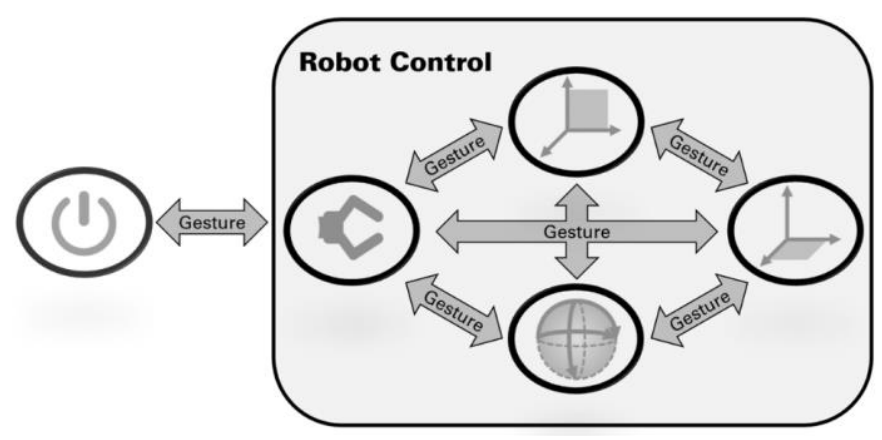

Fig. 11 Using the gesture based interface, the end-user can switch between the control groups. Start and stop of the robot control is enabled by head gestures.

\section{ELSI- ETHICAL, LEGAL AND SOCIAL IMPLICATIONS IN THE CONTEXT OF HUMAN-ROBOT-COLLABORATION}

\subsection{Fundamentals of ethical, legal and social implications}

The increasing use of collaborative robots in production, such as the use in assembly tasks, and assistive robots such as robots assisting people with disabilities, has advantages and disadvantages. On the one hand, working persons can be supported in physically strenuous or monotone tasks. On the other hand, the growth of robotic systems may cause jobs losses and a decline of human skills and knowledge. This dualism of technology leads to the necessity of considering an ethical point of view in the development of human-robot collaboration systems. Such questions belong to the field of technology ethics, an applied and problem-centered field which concentrates on normative uncertainties when dealing with technology. It includes moral questions of equipment and tool production and the use of technology [31]. Technology ethics reflects on design and impact of technology. Therefore, it is not concerned with technology itself, but with technology in the context of application. This reflection includes three criteria: aims and purposes of technology implementation (for example the support of humans at work by robots), instruments and means which are necessary for the application of technology (for example materials for manufacturing a robot and location of production), and consequences that arise by using the technology (for example risks of production for society and environment). Examining these criteria, technology ethics specifically focuses on the moral aspects involved. Besides, they also 
relate to scientific and technical progress. Additionally, technology ethics consider and integrate alternative options [32]. An important aspect concerning technology ethics is the concept of safety. The absence of unacceptable risks and dangers for humans should be guaranteed with the operation of the system [33] and the so called „safety” [34] must be created. Safety can be subdivided into two aspects: (1) the systems must not cause any danger to human beings and (2) the systems themselves and the data they contain must be protected against misuse [33].

Further, technology ethics deals with the usage and promotion of new opportunities that are enabled by technology. This will be shown on the example of the MeRoSy research project, which involves two scenario applications, robotic assistance of people with physical disabilities and robotic support of human employees in their work in industry. Due to the transformation from robots that are spatially and temporally separated from the working person and are programmed to execute tasks in a deterministic manner towards cooperative, adaptive lightweight robots, ethical, legal, and social implications (ELSI) should be considered [35].

\subsection{Method}

The purpose of this section is to determine how human-robot collaboration impacts technology. To this end potential ELSI problems and their possible causes and effects are identified and quantitatively analyzed based on the Aachen Model of Identification, Classification and Analysis of ethical, legal, and social Implications (AMICAI). Furthermore, the impacts of technology are identified and evaluated alongside potential risks, opportunities, and potentials of human-robot cooperation. The consideration of ethical, legal and social issues was carried out as follows: (1) identification of ethical, legal and social implications in several expert workshops, (2) classification of the implications by using the model for ethical evaluation of socio-technical arrangements (MEESTAR) [36], structured by stakeholder perspective and evaluation criteria, (3) quantitative analysis and prioritization of problems, and (4) development of solutions to address the causes of the problems [37][38].

To address the ELSI problems that could result from the installation of human-robotcooperative workplaces, and the resulting change in work, several expert workshops were conducted. The workshops were particularly regarding both collaborative scenarios (assistance of people with physical disabilities and support of employees in industrial assembly) of the MeRoSy project. The expert committee consists of MeRoSy Consortium Partners and Advisory Board as well as of further specialists (for example automation, robot gripper and robot manufacturers, system integrators, universities, end users of manufacturing systems and cooperative robot workplaces as well as of representatives of employers, social partners, and people with disabilities).

In a MeRoSy kick-off workshop general ethical, legal, and social questions were discussed in terms of the technical consequences of human-robot cooperation. Here, open questions and potential barriers as well as previous practical experiences were debated. In following workshops ELSI questions in the context of human-robot cooperation were identified, summarized and discussed in more depth.

After a consolidation of the ELSI problems gathered in the workshops, the ethical, legal and social aspects were divided according to the principle of the model for the ethical evaluation of socio-technical arrangements into three stakeholder perspectives (individual, organizational and societal) and seven ethical evaluation criteria (care, self-determination, safety, equality, privacy, participation and self-conception). The goal of this is to identify 
and classify the eventual ethical reservations and doubts when installing and using humanrobot-cooperative workplaces early on [36]. Thus, open questions can already be considered in the requirements analysis during the research and development process with design recommendations.

\subsection{Results}

In the following, the results of applying AMICAI based on expert workshops focusing on the application example of a human-robot cooperation workplace are summarized [37][ 38]. The expert committee named possible problem areas as well as reservations but also identified chances for the use of human-robot-cooperation workplaces in a structured dialogue, which they then evaluated and classified based on the criteria and planes of the MEESTAR-model.

With regard to the criterion of care, the experts saw the installation of human-robotcooperation workplaces as a challenge as well as an opportunity to improve work safety in which way human-robot collaboration could improve the wellbeing of the working person. On an organizational level, the employer is obligated to take measures to ensure and improve the health and safety of the employees. Further, installing new workplaces provides a chance to improve the ergonomics.

By contrast, there were mainly concerns with regard to the criterion of self-determination. For example, on an organizational level, it needs to be evaluated if the percentage of selfdetermined work (pace, etc.) is reduced due to the installation of human-robot-collaboration workplaces and the resulting revision of work processes.

Furthermore, ethical questions about the safety of the workplaces were raised. It needs to be cleared how the practical implementation of human-robot-collaboration can be done up-to-date regarding laws and guidelines.

More ethical doubts were expressed for the point of equality. In the assembly scenario it needs to be clarified if and to what extent a robot is allowed to replace a person. Also, on an individual level, the fear an employee has of a possible job loss needs to be taken seriously. In the other scenario, the assistance of physically disabled people, there are opportunities to reintegrate the people to work.

For the evaluation criterion of privacy, several possible problem areas were also diagnosed. The protection of an employee's data plays a particularly important role. Hence, on an organizational level, it should be discussed what data is analyzed and stored by human-robotworkplaces. This could lead to a conflict between employer and employee if the employee feels monitored and controlled by the robot.

In the area of participation, possibilities for the cooperation between people and robots were identified. The capacity to work can be retained for older people with the assistance of robots, while people that are already in retirement or physically handicapped people can be reintegrated. Furthermore, there are chances of job enlargement on an individual level. On the other hand, the danger exists that the share of low-skilled work is restricted [39].

Several conflict and problem areas were also identified for the criterion of self-conception. For example, it should be investigated if stress caused by the collaboration with robots or the fear of robots can lead to excessive demand on the worker or even a refusal to work or sabotage. Moreover, it needs to be tested to what degree the willingness to work together with a robot is dependent on the expected intuitive behavior (e.g. anthropomorphic movement) of the 
robot. Further, the means of interaction and communication with the robot needs to be clarified (e.g. visual, verbal, tactile) and what sensors (e.g. 3D-cameras) and aggregation, analysis and use of data fulfil the requirements of the protection of privacy.

\section{CONCLUSION AND FUTURE WORK}

In this paper, two human-robot cooperative scenarios representing different frameworks for human-robot interaction have been presented. The first scenario relates to the robot's cooperative work with an able bodied person in an industrial assembly while the second one relates to the robot's cooperative work in a library scenario with a person with disability. Due to different physical abilities of the human co-worker in the presented scenarios different humanrobot interfaces were developed and implemented. The human-robot cooperation in the second presented application scenario offers the opportunity for people with disabilities to re-enter into working life. With the presented head motion based interfaces, these people are able to control a robot without the need of their hands

The kinesthetic teaching used in the first human-robot cooperative scenario, industrial assembly scenario, is one of the possible human-robot interfaces for the able-bodied human demonstration of the task to be performed by the robotic co-worker. However, presented robot learning framework is a generic framework which can utilize different interfaces as the data acquisition module records the same data, the robot's joints angles and the end effector pose, independently of the interfaces. One of the future goals in MeRoSy project is to use the head motion-based robot control developed in the second presented scenario, assistive robot scenario, as the interface for the human demonstration of the task to be performed by the robot in the assembly scenario. Additionally, during the online working phase, a reinforcement learning algorithm will be included which will enable the human co-worker to provide feedback to the robot and to correct its actions if needed. This feedback will be provided using the head gestures interface as it is supposed that the hands of the human co-worker are occupied as the human is performing required actions to perform the task in the assembly scenario.

In the future work on further development of head gesture-based control of assistive robot, there will be the possibility to call robot skills from a library with a steadily increasing number of skills. The use of such a semi-autonomous robot will simplify the human-robot cooperation. Nevertheless, the user has a superior perception and thus has always the possibility to intervene and use the described direct control for fine-tuning of robot movements and for the correction in case of failure.

Beside the consideration of the human-robot cooperative technologies in two MeRoSy scenarios, this paper considered also the identification and classification of the ethical, legal and social implications (ELSI) in the context of human-robot cooperation. The contemplation of ethical, legal and social implications in the context of human-robot-cooperation shows what reservations and doubts can arise during the use of a socio-technical system. These ethical, legal and social implications were identified and classified with the help of the MEESTAR-model in MeRoSy expert workshops.

In the future, suitable measures and solutions need to be worked out after the identification and classification of ELSI. To this end, the methodical approach for the quantitative analysis of ethical, legal and social implication according to [37] is useful. Here the effect of the ethical, legal and social implications on the both MeRoSy scenarios will be considered. 
Acknowledgement: The research was supported by the German Federal Ministry of Education and Research (BMBF) as part of the project MeRoSy (Human Robot Synergy).

The authors thank MeRoSy industrial project partners for all the support in presented work, pi4 robotics GmbH for the technical support in assembly scenario and Vorwig GmbH for establishing safety concepts in both scenarios.

\section{REFERENCES}

[1] Gräser A., Heyer T., Fotoohi L., Lange U., Kampe H., Enjarini B., Heyer S., Fragkopoulos C., RistićDurrant D., "A Supportive FRIEND at Work: Robotic Workplace Assistance for the Disabled, " Robotics \& Automation Magazine, IEEE, vol.20, no.4, pp.148-159, Dec. 2013. [Online]. Available: http://dx.doi.org/ 10.1109/MRA.2013.2275695

[2] Slavnić S., Ristić-Durrant D., Tschakarow R., Brendel T., Tüttemann M., Leu A., Gräser A., "Mobile robotic gait rehabilitation system CORBYS - overview and first results on orthosis actuation, " in 2014 IEEE/RSJ International Conference on Intelligent Robots and Systems (IROS 2014), Chicago, USA, 2014. [Online]. Available: http://dx.doi.org/10.1109/IROS.2014.6942842

[3] "Innovative cooperation between workers and robots at Mercedes-Benz" [Online]. Available: http://robohub.org/innovative-cooperation-between-workers-and-robots-at-mercedes-benz-2/

[4] Ober-Gecks A., Henrich D. and Zwicker M., "Efficient GPU Photo Hull Reconstruction for Surveillance," in Proceedings of the International Conference on Distributed Smart Cameras, New York, Ny, USA, 2014. [Online] Available: http://dx.doi.org/ 10.1145/2659021.2659064

[5] Argall B. D., Chernova S., Veloso M., \& Browning B. "A survey of robot learning from demonstration," Robotics and Autonomous Systems, vol. 57, no. 5, pp. 469-483, May 2009. [Online] Available: http://dx.doi.org/ 10.1016/j.robot.2008.10.024

[6] Tellaeche A., Maurtua I., \& Ibarguren A. "Human robot interaction in industrial robotics. Examples from research centers to industry," in 2015 IEEE 20th Conference on Emerging Technologies \& Factory Automation (ETFA). Luxembourg, 2015. [Online] Available: http://dx.doi.org/10.1109/ETFA.2015.7301650

[7] "MeRoSy Project" [Online]. Available: http://www.technik-zum-menschen-bringen.de/projekte/merosy

[8] "pi4 Workerbot3 - Two-armed industrial robot with integrated image processing" [Online]. Available: http://www.pi4.de/fileadmin/material/datenblatt/Datenblatt_WB3_EN_V1_2.pdf

[9] Lee A., Gupta A., Lu H., Levine S. and Abbeel P., "Learning from multiple demonstrations using trajectory-aware non-rigid registration with applications to deformable object manipulation," in 2015 IEEE/RSJ International Conference on Intelligent Robots and Systems (IROS), Hamburg, 2015. [Online]. Available: http://dx.doi.org/10.1109/IROS.2015.7354120

[10] Schou C., Damgaard J., Bogh S. and Madsen O., "Human-robot interface for instructing industrial tasks using kinesthetic teaching," in 2013 44th International Symposium on Robotics, Seoul, 2013. [Online]. Available: http://dx.doi.org/10.1109/ISR.2013.6695599

[11] van Asseldonk E.H.F, Veneman J.F., Ekkelenkamp R., Buurke J. van der Helm F.C.T., van der Kooij, H. "The Effects on Kinematics and Muscle Activity of Walking in a Robotic Gait Trainer During Zero-Force Control," IEEE Transactions on Neural Systems and Rehabilitation Engineering, vol: 16, no: 4, Aug. 2008. [Online]. Available: http://dx.doi.org/10.1109/TNSRE.2008.925074

[12] Fang Q., "Reliable 3D Point Cloud Based Object Recognition" MSc thesis, University of Bremen, 2016

[13] "Kinect for Xbox One" [Online]. Available: http://www.xbox.com/en-US/xbox-one/accessories/kinect

[14] Reiner B., Ertel W., Posenauer H., and Schneider M., "LAT: A simple Learning from Demonstration method," in 2014 IEEE/RSJ International Conference on Intelligent Robots and Systems (IROS 2014), Chicago, IL, 2014, pp. 4436 - 4441. [Online]. Available: http://dx.doi.org/10.1109/IROS.2014.6943190

[15] Salzer H.E., "Formulas for best extrapolation," Numerische Mathematik, vol. 18, no. 2, pp. 144-153, April 1971. [Online]. Available: http://dx.doi.org/10.1007/BF01436323

[16] Sakoe H. and Chiba S., "Dynamic programming algorithm optimization for spoken word recognition," IEEE Transactions on Acoustics, Speech and Signal Processing, vol. 26, no. 1, pp. 43 - 49, 1978. [Online]. Available: http://dx.doi.org/10.1109/TASSP.1978.1163055

[17] Hsu Y.-L., C.-L., Tsai Y.-J., and Wang J.-S., "An Inertial Pen With Dynamic Time Warping Recognizer for Handwriting and Gesture Recognition," IEEE Sensors Journal, vol. 15, no. 1, pp. 154 - 163, 2014. [Online]. Available: http://dx.doi.org/10.1109/JSEN.2014.2339843

[18] Ramer U., "An iterative procedure for the polygonal approximation of plane curves," Computer Graphics and Image Processing, vol. 1, no. 3, pp. 244-256, 1972. 
[19] Douglas D. and Peucker T., "Algorithms for the reduction of the number of points required to represent a digitized line or its caricature," The Canadian Cartographer, vol. 10, no. 2, pp. 112-122, 1973

[20] Yu J., Gang C., Xiao Z., Wenlong C., and Yingxia P., "An improved Douglas-Peucker algorithm aimed at simplifying natural shoreline into direction-line," in 2013 21st International Conference on Geoinformatics (GEOINFORMATICS), Kaifeng, 2013, pp. 1 - 5. [Online]. Available: http://dx.doi.org/10.1109/ Geoinformatics.2013.6626177

[21] "ROS Wiki - rviz," [Online]. Available: http://wiki.ros.org/rviz

[22] "MoveIt - ROS," [Online]. Available: http://moveit.ros.org/

[23] Hillcrest Laboratories, Inc., "Hillcrest Labs - Experts in Motion," [Online]. Available: http://hillcrestlabs.com/ products/fsm-9/.

[24] Universal Robots A/S, "UR5 robot" [Online]. Available: http://www.universal-robots.com/products/ur5-robot/.

[25] Robotiq, "Adaptive Robot Gripper 2-Finger 85," [Online]. Available: http://robotiq.com/products/ industrial-robot-gripper/.

[26] Rudigkeit N., Gebhard M. und Gräser A., "Towards a User-Friendly AHRS-Based Human-Machine Interface for a Semi-Autonomous Robot," in 2014 IEEE/RSJ International Conference on Intelligent Robots and Systems, September 2014.

[27] Rudigkeit N., Gebhard M. und Gräser A., "Evaluation of Control Modes for Head Motion-based Control with Motion Sensors," 2015 IEEE International Symposium on Medical Measurements and Applications (MeMeA) - IMU Workshop, 2015. [Online]. Available: http://dx.doi.org/10.1109/MeMeA.2015.7145187.

[28] Rudigkeit N., Gebhard M. und Gräser A., "An Analytical Approach for Head Gesture Recognition with Motion Sensors," International Conference on Sensing Technology (ICST 2015), 2015.

[29] Rudigkeit N., Gebhard M. und Gräser A., "A Novel Interface for Intuitive Control of Assistive Robots Based on Inertial Measurement Units," in Ambient Assisted Living, Springer International Publishing, pp. 137-146, 2016.

[30] Jackowski A., Gebhard M. und Gräser A., "A Novel Head Gesture Based Interface for Hands-free Control of a Robot," in 2016 IEEE International Symposium on Medical Measurements and Applications (MeMeA), 2016. [Online]. Available: http://dx.doi.org/ 10.1109/MeMeA.2016.7533744

[31] Bendel O., "Die Industrie 4.0 aus ethischer Sicht", HMD Praxis der Wirtschaftsinformatik, vol. 52, no. 5, pp. 739-748, 2015. [Online]. Available: http://dx.doi.org/10.1365/s40702-015-0163-z

[32] Grunwald A., Handbuch Technikethik, J.B. Metzler, Stuttgard, 2013. [Online]. Available: http://dx.doi.org/10.1007/978-3-476-05333-6.

[33] Kagermann H., Wahlster W. \& Helbig J., "Umsetzungsempfehlungen für das Zukunftsprojekt Industrie 4.0: Abschlussbericht des Arbeitskreises Industrie 4.0.", Berlin, München: Forschungsunion im Stiftverband für die Deutsche Wissenschaft, 2013.

[34] Liggesmeyer P. and Trapp M., "Safety: Herausforderungen und Lösungsansätze", In T. Bauernhansl , M. ten Hompel \& B. Vogel-Heuser (Ed.), Industrie 4.0 in Produktion, Automatisierung und Logistik, pp. 433-449. Springer Vieweg, Wiesbaden, 2014. [Online]. Available: http://dx.doi.org/10.1007/978-3-658-04682-8_21

[35] Nelles J., Bröhl C., Spies J., Brandl C., Mertens A., Schlick C., "ELSI-Fragestellungen im Kontext der Mensch-Roboter-Kollaboration", In: Arbeit in komplexen Systemen - Digital, vernetzt, human?! Bericht zum 62. Arbeitswissenschaftlichen Kongress, Gesellschaft für Arbeitswissenschaft e.V. (GfA), GfA-Press, Dortmund, 2016

[36] Manzeschke A., Weber K., Rother E. \& Fangerau H.. "Ergebnisse der Studie Ethische Fragen im Bereich Altersgerechter Assistenzsystemen". 2013. [Online]. Available: http://www.mtidw.de/grundsatzfragen/ begleitforschung/dokumente/ethische-fragen-im-bereich-altersgerechter-assistenzsysteme-1.

[37] Wille M., Brandl C., Nelles J., Mertens A., Schlick C., "AMICAI: Ein methodisches Vorgehen zur quantitativen Analyse von ethischen, rechtlichen und sozialen Auswirkungen anwendungsnaher Forschungsprojekte, " In: Arbeit in komplexen Systemen - Digital, vernetzt, human?! Bericht zum 62. Arbeitswissenschaftlichen Kongress, Gesellschaft für Arbeitswissenschaft e.V. (GfA), GfA-Press, Dortmund, 2016.

[38] Nelles J., Kohns S., Spies J., Bröhl C., Brandl C., Mertens A., \& Schlick C. M., "Best-practice approach for a solution-oriented technology assessment: Ethical, legal, and social issues in the context of humanrobot collaboration." In Advances in Ergonomic Design of Systems, Products and Processes, Springer, Berlin Heidelberg, 2017 (in print).

[39] Dworschak B., "Kompetenzen der Zukunft in der Industrie 4.0 - Anwendungsfälle, Szenarien, Entwicklungspfade. In Gesellschaft für Arbeitswissenschaft e.V. (GfA) (Hrsg.) ", in VerANTWORTung für die Arbeit der Zukunft (S. 1-5). Bericht zum 61. Arbeitswissenschaftlichen KongressGesellschaft für Arbeitswissenschaft e.V. (GfA), GfA-Press, Dortmund, 2015. 